\title{
The effects of autobiographical elaboration on noun recall
}

\author{
MICHAEL W. WARREN, DAVID CHATTIN, D. DAVID THOMPSON, \\ and MARYANN T. TOMSKY \\ Randolph-Macon College, Ashland, Virginia
}

\begin{abstract}
Four experiments demonstrate that autobiographical elaboration is a rich and powerful encoding process. Autobiographical elaboration was induced by using a modified free-association procedure. Subjects were presented stimulus words and were instructed to think of a personal experience that the words reminded them of and to date the remembered experience on a 7-point temporal-category scale (i.e., minutes, hours, days, weeks, months, years, never). Unexpected free recall of the stimulus words showed a recency effect (Experiments 1, 3, and 4), autobiographical orienting produced better noun recall than either a semantic or a structural orienting task (Experiments 2 and 3), and the recency effect observed in Experiments 1, 3, and 4 appears to have been due to differences in autobiographical elaboration (Experiment 4).
\end{abstract}

Crovitz and Schiffman (1974), Galton (1911), and Robinson (1976) have all used a modified free-association procedure to investigate autobiographical memory. Subjects were presented stimulus words and were instructed to think of an experience from their lives of which the word reminded them and to date the remembered experience. These experimenters investigated the relationship between different stimulus words (e.g., nouns, adjectives, verbs) and qualities of the corresponding recollections (Galton, 1911; Robinson, 1976) and the frequency of recollections as a function of their ages (Crovitz \& Schiffman, 1975).

Nesbitt and Allen (Note 1) used a similar technique, but required unexpected free recall of the stimulus words. They read a list of low-frequency nouns to students and asked the students to estimate, for each noun, how long it had been since their last perceptual contact with the object named by the noun. Subjects were asked to report each estimate in one of seven categories: minutes, hours, days, weeks, months, years, and never. The results showed that incidental recall was an inverse function of temporal category (i.e., a recency effect). Presumably, the students estimated how long it had been since their last perceptual contact with the objects by recalling autobiographical episodes associated with the nouns and dating the episodes. Thus, autobiographical elaboration may have produced the interest-

These experiments were conducted during independent studies by the junior authors under the direction of the first author. This research was supported in part by equipment acquired under NSF ISEP Grant SER-8013303 to the first author. Portions of this research were presented at the 1980 Southeastern Psychological Association Meeting and the 1980 and 1981 Carolinas Psychology Conference. Requests for reprints should be sent to Michael W. Warren, IH-135, AT\&T Bell Laboratories, Inc., 6200 East Broad Street, Columbus, Ohio 43213. ing recency effect observed by Nesbitt and Allen (Note 1).

The concept of autobiographical elaboration helps explain some levels-of-processing research. In particular, consider the results of Packman and Battig (1978) and Rogers, Kuiper, and Kirker (1977).

Packman and Battig (1978) compared recall and recognition for seven semantic orienting tasks: rating memory items on a seven-point scale of pleasantness, concreteness, imagery, categorizability, meaningfulness, familiarity, and number of attributes. Their data showed that pleasantness was significantly superior to all other dimensions. One interpretation of this result is that rating pleasantness produced additional elaboration not inherent in the other semantic rating tasks. That is, rating the pleasantness of a word encouraged subjects to process the meaning of the word and to retrieve autobiographical memories associated with the word. For example, whereas one subject may judge "dog" to be "very unpleasant" because he or she has been bitten several times, another subject may judge "dog" to be "pleasant" because he or she has fond memories of a childhood pet. In both cases, autobiographical memories are considered in making the pleasantness judgment, and the retrieval of autobiographical memories should provide additional elaboration and improve memorability. It seems unlikely that autobiographical memories would be elicited to the same degree in rating words on the other dimensions.

Rogers et al. (1977) reported that self-reference is "a rich and powerful encoding process." In their experiments, subjects rated adjectives in four tasks designed to force different kinds of encoding: structural(Big letters?), phonemic (Rhymes with XXXX?), semantic (Means same as YYYY?), and self-reference (Describes you?). Adjectives rated under the self-reference task were recalled best. Rating whether an adjective describes you 
requires processing the meaning of the adjective and should elicit autobiographical memories. One would be unlikely to rate him- or herself as "shy" if he or she could not remember behaving "shyly." Again, the retrieval of autobiographical memories associated with the adjectives should produce additional elaboration and improve recall relative to the other conditions.

The present paper reports four experiments that investigated autobiographical elaboration. Experiments 1,2 , and 4 demonstrated that object-label recall is an inverse function of rated recency of perceptual contact with the objects. Experiments 2 and 3 demonstrated that autobiographical orienting produces better noun recall than either a semantic or a structural orienting task. Experiment 4 suggested that the recency effect observed in Experiments 1, 2, and 4 was due to differences in autobiographical elaboration.

\section{EXPERIMENT 1}

Experiment 1 was, in part, a replication of a phenomenon reported by Nesbitt and Allen (Note 1). They read a list of low frequency nouns to students, and asked the students to estimate, for each noun, how long it had been since their last perceptual contact with the object referred to by the noun. Subjects were asked to report each estimate in one of seven categories (i.e., minutes, hours, days, weeks, months, years, never). Unexpected free recall was an inverse function of temporal category (i.e., a recency effect).

We considered two explanations for the result. One explanation is that recency of perceptual contact may be a word attribute, like concreteness, that is related to recall. We could speculate that the memorial representation of an object label may be enhanced (i.e., show an increase in strength or accessibility) by recent perceptual contact with the object. Thus, ratings of recency of perceptual contact may indirectly reflect differences in the memorability of words.

A second explanation is that rating the recency of perceptual contact is an orienting task that produces differences in encoding elaboration. That is, rating how long it has been since perceptual contact with objects named by nouns may encourage subjects to retrieve and date autobiographical memories associated with the nouns. If rated recency of perceptual contact and the likelihood of autobiographical elaboration are inversely related (i.e., words assigned to the "minutes" category receive more autobiographical elaboration than words assigned to the "months" category), then the Nesbitt and Allen (Note 1) result would obtain.

In order to test these competing explanations, we must consider how word attributes and orienting tasks influence recall. It is not necessary to focus attention on the dimensions of concreteness or Thorndike-Lorge (1944) frequency via some orienting task (e.g., rating words on a seven-point scale of concreteness or familiarity) for these attributes to influence recall. Indeed, if subjects were to judge the concreteness of words before recall or even after recall, their recall scores would correlate with their concreteness ratings in both instances. By analogy, if recency of perceptual contact is a word attribute, it should influence recall in the absence of orienting instructions focusing attention on the dimension, and recall should be an inverse function of both prerecall and postrecall rated recency of perceptual contact.

Orienting tasks influence encoding by focusing attention on a particular aspect of the memory stimulus, an aspect that is not typically processed. It is unlikely that subjects process the degree of pleasantness or selfreference of words unless they are given instructions that encourage such processing. Thus, to assert that the inverse relationship between recall and rated recency of perceptual contact is an orienting-task effect, implies that prerecall ratings, but not postrecall ratings will be related to recall.

The appropriate comparison to test these competing explanations is to compare the relationship between incidental recall and temporal category for two conditions: one in which subjects rate recency of perceptual contact before recall, and one in which subjects rate recency of perceptual contact after recall. If the Nesbitt and Allen (Note 1) result represents a word-attribute effect, recall for both conditions should be inversely related to temporal category, and a main effect of temporal category should obtain. However, if the Nesbitt and Allen result represents an orienting-task effect, then the recall of the prerecall, but not the postrecall rating condition should be inversely related to temporal category, and an interaction of rating condition and temporal category should obtain.

\section{Method}

Students in each of two introductory psychology classes were assigned randomly (FORTRAN algorithm) to one of two conditions: (1) prerecall rating of recency of perceptual contact ( $n=18: 7$ males, 11 females), hereafter referred to as Pre-RPC, and (2) postrecall rating of recency of perceptual contact $(n=18$ : 6 males, 12 fermales), hereafter referred to as Post-RPC. Two male subjects were eliminated randomly from the original PostRPC group to balance sex and number of subjects across conditions. Subjects who failed to use one of the first six temporal categories were eliminated to achieve equal numbers of subjects across conditions and temporal categories; 1 female subject was eliminated from the Post-RPC condition for failing to use the "minutes" category.

A group procedure was used. The subjects participated in the experiment during regularly scheduled classes, with approximately one-half of the class assigned to each conditon. Each subject received a computer-generated five-page booklet (FORTRAN algorithm) with instructions and an example of how the orienting task was to be performed on the first page. Fourteen stimuli were printed vertically on the second page, 15 stimuli were printed on the next two pages, and 6 stimuli were printed on the last page. The appropriate rating scale appeared to the right of each stimulus.

The subjects rated the words at their own pace. They were told that the experimenters were collecting normative data; no indication was given that recall would be required later. Following the rating task, the rating booklets were collected and recall 
sheets were distributed. This took approximately $3 \mathrm{~min}$. Thus, the subjects experienced retention intervals of varying lengths (approximate range: 3 to $10 \mathrm{~min}$ ), depending on how quickly they finished the rating task. Eight minutes were allowed for written free recall.

The Pre-RPC subjects rated words on a 7-point recency-ofperceptual-contact scale (1-minutes, 2-hours, 3-days, 4weeks, 5-months, 6-years, 7-never). They received the following orienting-task instructions: "You are to rate each of the words printed below by circling the number that indicates how long it has been since your last visual contact with the object named by the word. That is, indicate when you last saw the object or a picture of the object. This task is best accomplished by thinking about the object until you can remember a personal episode involving the object or a picture of the object. Then, decide when the episode occurred and circle the appropriate number."

In order to match the prerecall activities of the two conditions as closely as possible, the Post-RPC subjects performed a "neutral" control orienting task before recall. They rated words on a 5-point scale of memorability (1-very unlikely, 2-unlikely, 3-uncertain, 4-likely, 5-very likely) and received the following prerecall orienting-task instructions: "You are to rate each of the words printed below by circling the number that indicates how likely you would be to recall the particular word if you were asked to recall these words two days from now. That is in two days would you be unlikely to recall the word, are you uncertain whether you would recall it, or are you very likely to recall the word." Following recall, the Post-RPC subjects completed the recency-of-perceptual-contact rating task on the same words.

Subjects in both conditions received a different random ordering (FORTRAN algorithm) of the same 50 low ThorndikeLorge frequency $($ mean $=5.92$ ) concrete nouns $(C>6.00)$. The words were chosen to represent a wide variety of objects, animals, and occupations and to have recall indexes of 344 or higher in the Christian, Bickley, Tarka, and Clayton (1978) norms.

\section{Results and Discussion}

The proportions correct under temporal categories were subjected to a 2 (rating condition) $\times 6$ (temporal category) mixed analysis of variance. The "never" category was not included in the analysis because 8 of the 36 subjects failed to rate words in the "never" temporal category. However, words rated in the first six temporal categories accounted for $95 \%$ and $94 \%$ of the Pre- and Post-RPC ratings, respectively. The analysis revealed a significant main effect of rating condition $[F(1,34)=11.37$, MSe $=.139, p<.01]$, a significant main effect of temporal category $[F(5,170)=10.46$,
MSe $=.046, p<.001]$, and a significant interaction of rating condition $x$ temporal category $[F(5,170)=4.30$, MSe $=.046, p<.01]$. The results are summarized in Table 1.

The Pre-RPC condition showed better recall than the Post-RPC condition (mean proportions correct were .556 and .384 , respectively). The superior recall in the Pre-RPC condition suggests that the recency-ofperceptual-contact orienting instructions produced more elaborate encoding. Presumably, autobiographical memories were retrieved and considered in making the temporal judgments involved in rating recency of perceptual contact, and the retrieval of these autobiographical memories prompted a more elaborate encoding of memory items. It seems unlikely that the Post-RPC condition prerecall orienting instructions (rating likelihood of recall) would elicit autobiographical memories to the same degree

It is also possible that the difference in memory performance for the Pre- and Post-RPC conditions reflects differences in study times. That is, making temporal judgments on a 7-point scale may require more time than making memorability judgments on a 5-point scale, and this additional processing time may account for the difference in recall performance. This potential confound was addressed in Experiment 3. For now, it is sufficient to note that other researchers have failed to find a positive relationship between depth of processing and processing time (see Craik \& Tulving, 1975, Eysenck \& Eysenck, 1979, and Rogers et al., 1977).

The Pre-RPC condition showed a memory curve similar to the one reported by Nesbitt and Allen (Note 1), but clearly different from the Post-RPC condition (see Table 1). Indeed, tests for simple effects revealed an effect of temporal category for the Pre-RPC condition $[F(5,85)=13.55, \mathrm{MSe}=.046, p<.001]$, but no effect of temporal category for the Post-RPC condition $[\mathrm{F}(5,85)=1.44, \mathrm{MSe}=.046, \mathrm{p}>.10]$. Note that the interaction of rating condition $x$ temporal category is an effect of judged recency, not of temporal recency. If the recency effect were due to the fact that higher frequency items tend to be encountered more recently and are better recalled, then we should have

Table 1

Mean Number of Words Rated and Mean Proportion of Words Recalled as a

Function of Rating Condition and Temporal Category in Experiment 1

\begin{tabular}{|c|c|c|c|c|c|c|c|}
\hline & \multicolumn{7}{|c|}{ Temporal Category } \\
\hline & Minutes & Hours & Days & Weeks & Months & Years & Never \\
\hline & \multicolumn{7}{|c|}{ Condition: Prerecall Rating of Recency of Perceptual Contact } \\
\hline \multirow{3}{*}{$\begin{array}{l}\text { Mean } \\
\text { Proportion }\end{array}$} & 3.83 & 4.83 & 10.78 & 8.33 & 11.67 & 7.94 & 2.61 \\
\hline & .856 & .709 & .396 & .490 & .433 & .449 & \\
\hline & \multicolumn{7}{|c|}{ Condition: Postrecall Rating of Recency of Perceptual Contact } \\
\hline Mean & 4.39 & 5.72 & 10.28 & 8.55 & 10.17 & 7.89 & 3.00 \\
\hline Proportion & .439 & .468 & .392 & .318 & .374 & .319 & \\
\hline
\end{tabular}


observed a recency effect for both rating conditions and a main effect of temporal category. On the basis of these results, the Nesbitt and Allen (Note 1) result appears to be an orienting-task effect.

A potential confound must be dismissed if we are to be confident of an orienting-task-effect explanation of these results. One could argue that the interaction of rating condition $x$ temporal category is an artifact of the way in which the Pre- and Post-RPC subjects rated recency of perceptual contact. That is, the subjects in the Post-RPC condition may not have given accurate temporal ratings because they rushed through the second rating task and devoted little, if any, attention to rating words on the recency-of-perceptual-contact dimension. This argument would receive some support if the distributions of recency-of-perceptual-contact ratings were significantly different for the Pre- and Post-RPC conditions. To this end, the frequencies of items rated under temporal categories were subjected to a 2 (rating condition) $\times 7$ (temporal category) mixed analysis of variance. The analysis revealed a significant main effect of temporal category $[\mathrm{F}(6,204)=26.49, \mathrm{MSe}=13.46, \mathrm{p}<.001]$; however, the effect of rating condition $[F(1,34)<1]$ and the interaction of rating condition $\mathrm{x}$ temporal category $[F(6,204)<1]$ were not significant. The results (see Table 1) showed that the two distributions were quite similar. Thus, the interaction of rating condition $\mathrm{x}$ temporal category does not appear to be an artifact of the way in which the Pre- and Post-RPC subjects rated recency of perceptual contact.

Another question of some concern is the origin of the recency effect for the Pre-RPC condition. The recency effect may be limited to items rated in the "minutes" category, and many of these items may have been encountered in the experimental situation. If so, then their recall could be facilitated by environmental/ situational cues still present at the time of recall. For example, if one of the stimulus words were "pencil" and most subjects assigned "pencil" to the "minutes" category and recalled it, this would not be a surprising result because all of the subjects used a pencil during the experiment to rate the stimulus words. A recency effect caused solely by this phenomenon would not be particularly interesting.

Post hoc analysis (Newman-Keuls) of the Pre-RPC recall scores showed that the recall ordering of "minutes" $>$ "hours" and "hours" $>$ "days" were both significant $(p<.05)$, that the "minutes" and "hours" categories were significantly larger than all other temporal categories $(p<.05)$, and that none of the other differences were significant. An item analysis revealed that the following words were rated in the "minutes" and "hours" categories: abdomen, alcohol, alligator, ankle, basement, blister, bouquet, brassiere, goblet, headlight, keg, lemonade, locker, macaroni, mosquito, nun, pianist, poster, refrigerator, slush, skillet, skull, suds, tripod, tweezers, and whale. Only four of these words (i.e., abdomen, ankle, brassiere, and skull) are likely to have been encountered in our experimental situation. These words accounted for $12.4 \%, 14.1 \%, 10.6 \%$, and .8\%, respectively, of the items recalled in the "minutes" and "hours" categories. Thus, items that may have been encountered in the experimental situation accounted for $37.9 \%$ of the items recalled in the "minutes" and "hours" categories. On the other hand, three items that were not likely to have been encountered in the experimental situation (i.e., headlight, poster, and refrigerator) accounted for $35.4 \%$ of the items recalled in the "minutes" and "hours" categories. Clearly, the recency effect is not limited to the "minutes" category, and it is not entirely due to the recall of items that may have been encountered in the experimental situation. Thus, it seems unlikely that the recency effect is an artifact of subjects' noticing certain objects in the experimental environment named by the stimulus words and recalling these words more readily than other stimulus words.

In summary, using a different method of presentation and different stimuli, Experiment 1 replicated Nesbitt and Allen (Note 1). Recency-of-perceptualcontact instructions produced better recall than likelihood-of-recall orienting instructions. The relationship between recall and temporal category observed here and by Nesbitt and Allen (Note 1) appears to be an orientingtask effect.

\section{EXPERIMENT 2}

Experiment 1 showed that rating nouns for recency of perceptual contact produced better recall than rating nouns for likelihood of recall. Presumably, autobiographical memories were retrieved and considered in making many of the temporal judgments involved in rating recency of perceptual contact, and the retrieval of these autobiographical memories produced more encoding elaboration and better recall. This leads to questions concerning where the rating of recency of perceptual contact falls in relation to other orienting tasks.

You may recall from the introduction that Packman and Battig (1978) compared recall and recognition for seven semantic orienting tasks and found rating pleasantness to be superior to the other orienting tasks. We suggested that rating the pleasantness of a word increased the likelihood of retrieving autobiographical memories associated with the word, and that this additional autobiographical elaboration improved memorability. We would expect that rating the recency of perceptual contact would lead to more au tobiographical elaboration than would rating pleasantness, because the recency-ofperceptual-contact orienting-task instructions explicitly encourage the retrieval of autobiographical episodes. Of course, both the recency-of-perceptual-contact and the pleasantness orienting tasks should lead to more elaboration and better recall than a structural orienting task, such as rating the length of words (see Craik \& Tulving, 1975). To this end, we compared recall for 
words rated on recency of perceptual contact, pleasantness, and length. We anticipated that recall performance for the different orienting conditions would order as follows: recency of perceptual contact $>$ pleasantness $>$ length.

\section{Method}

Thirty-nine subjects from several undergraduate psychology classes participated in the experiment during regularly scheduled classes. They received a nine-page computer-generated booklet (FORTRAN algorithm) with instructions and examples of how the three orienting tasks were to be performed. Six nouns appeared on each of the remaining seven pages, for a total of 42 nouns (subset of Experiment 1 stimuli). For each set of three words, the subjects were required to rate a word on a 7 -point scale of recency of perceptual contact $(1-$ minutes, 2-hours, 3-days, 4-weeks, 5-months, 6-years, 7-never), a 7-point scale of pleasantness (1-very unpleasant, 4-neutral, 7 -very pleasant; $2,3,5$, and 6 were not labeled), or a 7-point scale of length (1-very short, 4 -average, 7 -very long; $2,3,5$, and 6 were not labeled). The appropriate rating scale appeared to the right of each stimulus.

The order of the rating tasks was randomized, each word appeared in each rating task equally of ten, and serial position was randomized (all via FORTRAN algorithm). The subjects rated the words at their own pace. They were told that the experimenters were collecting normative data; no indication was given that immediate recall would be required. Following the rating task, the rating booklets were collected and recall sheets were distributed. This took approximately $3 \mathrm{~min}$. Thus, the subjects experienced retention intervals of varying lengths (approximate range: 3 to $10 \mathrm{~min}$, depending on how quickly they finished the rating task. Six minutes were allowed for written free recall.

The instructions for the three orienting tasks were as follows: (1) Recency of perceptual contact-same as in Experiment 1. (2) Pleasantness - "Words vary as to whether they have a pleasant or unpleasant connotation. For instance, the word 'funeral' has unpleasant connotations, such as death, mourning, sorrow, etc. Thus, many of us would rate 'funeral' by circling ' 1 ' for 'very unpleasant.' So, words are rated on this dimension by circling the number that indicates the degree of pleasant ness/ unpleasantness suggested by the word." (3) Length-"Words vary in length or the number of letters comprising the word A two-letter word might be rated as ' 1 ' for 'very short' while a 12-1etter word might be rated as ' 7 ' for 'very long.' Thus, words are rated on this dimension by circling the number that indicates how long you feel the word is."

\section{Results and Discussion}

A one-way within-subjects analysis of variance revealed a significant effect of orienting task $[F(2,76)=$ $38.96, \mathrm{MSe}=3.58, \mathrm{p}<.001]$. The mean numbers of words recalled for the recency-of-perceptual-contact, pleasantness, and length-of-word orienting tasks were $6.92,5.53$, and 3.17 , respectively. Sign tests showed that rating the recency of perceptual contact produced better recall than did rating pleasantness $(Z=2.65, p<.01)$, a rating task that Packman and Battig (1978) had found produced better recall than six other semantic orienting tasks, and that rating pleasantness produced better recall than did rating length $(Z=4.77, p<.001)$. The results were consistent with an autobiographical elaboration hypothesis: The more an orienting task elicits autobiographical memories, the better recall will be. Of course, it is also possible that the differences in memory performance for the three conditions reflected differences in study times. Experiment 3 addressed this and other issues.

\section{EXPERIMENT 3}

In the first two experiments, we noted a potential confound of study time or rating time with autobiographical elaboration. That is, we suggested that making the temporal judgments involved in rating the recency of perceptual contact may require more time than making the judgments involved in rating likelihood of recall (Experiment 1) or pleasantness and length (Experiment 2), and that this additional study time may have caused the superior recall for recency-of-perceptualcontact orienting instructions that was observed in Experiments 1 and 2. In order to assess the effect of study time in this experiment, rating task (i.e., recency of perceptual contact, pleasantness, or length) was manipulated as a between-subjects factor and rating-task latencies were recorded. If rating time or study time is an important factor, its effect should be observed in the following ways: (1) Differences in recall performance across rating tasks should be accompanied by corresponding differences in rating times, and (2) within each rating task, subjects with longer rating times should demonstrate better recall. On the other hand, if qualitative differences in encoding elaboration produce the differences in recall, then recall should vary between rating tasks, but within any given task, recall should not depend on rating time (see Craik \& Tulving, 1975, p. 279).

Another question of some concern was whether our replication of Nesbitt and Allen's (Note 1) observation of an inverse relationship between recall and temporal category could be explained as an artifact of the distribution of rating responses. Consider the Pre-Recall condition in Table 1. It shows that rating frequency increases over the first three temporal categories and that recall declines over the same three temporal categories. It is important to note that, if subjects' rating frequencies increase with temporal category and they recall approximately the same number of words from each category, an inverse relationship between recall and temporal category must obtain. An example may help to clarify this point. If subjects rated 5,10 , and 15 words in the "minutes," "hours," and "days" temporal categories, and then recalled about 4 words from each temporal category, their performance would produce recall proportions of $.80, .40$, and .27 for the "minutes," "hours," and "days" categories, respectively. So, the inverse relationship between recall and temporal category may simply reflect the tendency of subjects to assign low Thorndike-Lorge frequency nouns to the later temporal categories (i.e., "days," "weeks," "months," "years"). This explanation would be contraindicated if we could alter the distribution of temporal category ratings and still observe the inverse relationship 
between recall and temporal category. One approach to achieving this result is to include high ThorndikeLorge frequency words among the memory stimuli to increase the likelihood of subjects' using the early temporal categories. If substantially more words were assigned to the early temporal categories (i.e., "minutes" and "hours"), and the inverse relationship between recall and temporal category still obtained, then the inverse relationship between recall and temporal category could not be explained as an artifact of the distribution of temporal ratings. To this end, ThorndikeLorge frequency was manipulated as a within-subjects factor in Experiment 3.

\section{Method}

Fifty-four subjects in two introductory psychology classes served in the experiment. Two subjects were eliminated: one at random to balance subjects across conditions, and one for failing to follow instructions. Sex was balanced across conditions.

The subjects were assigned randomly to one of three orienting tasks: recency of perceptual contact, pleasantness, or length. Each subject received a nine-page computer-generated booklet (FORTRAN algorithm) with instructions and an example of the orienting task on the first page. Three high Thorndike-Lorge frequency nouns and three low Thorndike-Lorge frequency nouns appeared on each of the remaining eight pages, for a total of 48 nouns. The nouns were all high-concrete $(C>6.00)$, high-imagery ( $\mathrm{I}>6.00$ ) nouns selected from the Paivio, Yuille, and Madigan (1968) norms. The 24 low-frequency nouns had a mean frequency of 4.5 occurrences per million; the 24 highfrequency nouns had a mean frequency of 88.79 occurrences per million (A and AA words were assigned 50 and 99, respectively). Serial position and the order of the high- and lowfrequency nouns on each page were randomized (FORTRAN algorithm).

A digital clock was placed at the front of the classroom so that the subjects could record the time they began and completed the rating task. Times were recorded to the nearest minute. The subjects were instructed that the rating task was not a speed test and that they should take their time and rate each word carefully. The remaining procedures were identical to those of Experiment 2.

\section{Results and Discussion}

The number of items recalled was subjected to a 3 (rating condition) $\times 2$ (Thorndike-Lorge frequency) mixed analysis of variance. The analysis revealed a significant main effect of rating condition $[F(2,51)=$ 63.89$, MSe $=9.94, p<.001]$, a significant effect of Thorndike-Lorge frequency $[\mathrm{F}(1,51)=37.85$, $\mathrm{MSe}=$ $4.66, p<.001]$, and a significant interaction of rating condition $x$ Thorndike-Lorge frequency $[F(2,51)=$

Table 2

Mean Number of Words Recalled as a Function of Rating

Condition and Thorndike-Lorge (T-L) Frequency in Experiment 3

\begin{tabular}{lcr}
\hline \multirow{2}{*}{ Rating Condition } & \multicolumn{2}{c}{ T-L Frequency } \\
\cline { 2 - 3 } \multicolumn{1}{c}{ High } & Low \\
\hline Recency of Perceptual Contact & 13.33 & 10.94 \\
Pleasantness & 11.06 & 7.11 \\
Length & 4.50 & 3.17 \\
\hline
\end{tabular}

$4.66, \mathrm{MSe}=4.66, \mathrm{p}<.05]$. The results are summarized in Table 2.

The interaction of rating condition $x$ ThorndikeLorge frequency indicates that at least two of the rating conditions were influenced to varying degrees by Thorndike-Lorge frequency. Comparisons were made between the recency-of-perceptual-contact and the pleasantness groups, the recency-of-perceptual-contact and the length groups, and the pleasantness and the length groups. The results showed a significant interaction of rating condition $x$ Thorndike-Lorge frequency for the pleasantness and length conditions $[F(1,34)=$ $7.99, \mathrm{MSe}=30.68, \mathrm{p}<.01]$; none of the other comparisons were significant. Although this result is not particularly important for our purposes, it shows that Thorndike-Lorge frequency had a larger effect on the pleasantness rating condition than on the length rating condition. The result suggests that the Thorndike-Lorge frequency effect is influenced by encoding operations.

The recall results replicated those of Experiment 2. The mean numbers of items recalled for the recency-ofperceptual-contact, the pleasantness, and the length rating conditions were $24.28,18.17$, and 7.67 , respectively. Post hoc tests (Newman-Keuls) showed that the recall ordering of recency of perceptual contact $>$ pleasantness $>$ length was significant $(p<.05)$ at both levels of Thorndike-Lorge frequency. This replication is important because it allows us to dismiss two potential explanations for the superior recall performance of recency-of-perceptual-contact orienting instructions that was observed in Experiments 1 and 2.

First, one could argue that the superior recall of recency-of-perceptual-contact orienting instructions in Experiment 2 resulted from interactive effects due to the concurrent use of multiple semantic dimensions. That is, the requirement to rate words on more than one dimension may have enhanced one dimension at the expense of another, thereby prompting more attention to words rated on that dimension, and better recall of those words. This explanation is contraindicated because the subjects in Experiment 3 rated words on a single dimension and produced the same recall results as in Experiment 2.

Second, one could argue that the superior recall for recency-of-perceptual-contact orienting instructions in Experiments 1 and 2 represent a list-specific result, because the same verbal stimuli were used in Experiments 1 and 2. This explanation is unlikely, because Experiment 3 showed the same result, but half of the stimuli were high-frequency words that were not used in Experiments 1 and 2.

The rating-task latencies were subjected to a one-way between-subjects analysis of variance. The analysis revealed a significant effect of rating task $[F(2,51)=$ 14.02, MSe $=3.05, \mathrm{p}<.001]$. The mean latencies in minutes for the recency-of-perceptual-contact, pleasantness, and length rating tasks were $7.17,4.44$, and 4.55 , respectively. Post hoc tests (Newman-Keuls) revealed that subjects took significantly longer to complete the 
recency-of-perceptual-contact rating task than any other rating task $(p<.05)$, and that the difference in rating times for the pleasantness and length rating tasks was not significant. This was an equivocal result. The superior recall of the recency-of-perceptual-contact condition was accompanied by a significantly longer mean rating time. However, the difference in mean rating times for the pleasantness and length conditions was not significant and was not in the correct direction to account for the significantly better recall of the pleasantness condition.

To analyze the effect of rating time within rating conditions, the subjects were divided into fast and slow raters via a median split, and their recall scores were analyzed via a 2 (rating speed: fast or slow) $\times 2$ (ThorndikeLorge frequency) $\times 3$ (rating condition) mixed analysis of variance. Mean rating times and recall scores are shown in Table 3.

$\mathrm{T}$ tests showed that the difference in mean rating times for the fast and slow raters were significantly different for all of the rating conditions ( $p<.001$ for all comparisons). Slow raters spent, on the average, $2.44 \mathrm{~min}(3.05 \mathrm{sec}$ per item) longer rating items in the length condition, $1.78 \mathrm{~min}(2.22 \mathrm{sec}$ per item) longer rating items in the pleasantness condition, and $3.67 \mathrm{~min}$ (4.59 $\mathrm{sec}$ per item) longer rating items in the recency-ofperceptual contact condition. Note that the magnitude

Table 3

Mean Rating Times as a Function of Rating Condition and Mean Words Recalled as a Function of Rating Condition and Thorndike-Lorge (T-L) Frequency for Fast and Slow Raters in Experiment 3

\begin{tabular}{lcccc}
\hline & \multicolumn{2}{c}{ Rating Times } & \multicolumn{2}{c}{ Recall } \\
\cline { 2 - 3 } Subjects & Minutes & Seconds/Item & High T-L & Low T-L \\
\hline \multicolumn{5}{c}{ Rating Condition: Length } \\
Fast & 3.33 & 4.16 & 5.33 & 3.33 \\
Slow & 5.77 & 7.21 & 3.66 & 3.00 \\
& Rating & Condition: Pleasantness \\
Fast & 3.55 & 4.44 & 10.77 & 6.55 \\
Slow & 5.33 & 6.66 & 11.33 & 7.66 \\
\multicolumn{1}{c}{ Rating Condition: Recency of Perceptual Contact } \\
Fast & 5.33 & 6.66 & 13.11 & 10.65 \\
Slow & 9.00 & 11.25 & 13.55 & 11.22 \\
\hline
\end{tabular}

of the difference in rating times between fast and slow raters in the length condition (2.44 min) and recency-ofperceptual-contact condition $(3.67 \mathrm{~min}$ ) is approximately equal to or larger than the magnitude of the difference in rating times between the recency-of-perceptualcontact and length conditions $(2.62 \mathrm{~min})$ and the recency-of-perceptual-contact and pleasantness conditions $(2.73 \mathrm{~min})$. Thus, if the difference in rating time produced the superior recall in the recency-of-perceptualcontact condition, then we would expect slow raters to recall more than fast raters in both the length and the recency-of-perceptual-contact conditions.

The analysis of recall scores showed significant main effects of rating condition $[\mathrm{F}(2,48)=62.29$, $\mathrm{MSe}=$ $635.28, p<.001]$ and Thorndike-Lorge frequency $[\mathrm{F}(1,48)=36.34, \mathrm{MSe}=176.33, \mathrm{p}<.001]$, but no effect of rating speed $[F(1,48)<1]$. The interaction of rating condition $x$ Thorndike-Lorge frequency was significant $[F(1,48)=3.20, \mathrm{MSe}=15.53, \mathrm{p}<.05]$; none of the other interactions were significant (all Fs $<1$ ). The important result for our purposes is that, although slow raters took significantly longer to rate items in all conditions, there were no significant effects of rating speed on item recall. This is in spite of the fact that the experimental procedure encouraged recall differences between fast and slow raters--fast raters spent less time on the average on each item, and since they finished sooner, had to wait longer to recall. Thus, the withincondition rating-time analysis weakens a rating-time or study-time interpretation of the recall data and reinforces the interpretation that the recall data were due to qualitative differences in elaboration produced by the various encoding tasks. In this vein, it is important to note that other researchers have failed to find a positive relationship between depth of processing and processing time (see Craik \& Tulving, 1975, Eysenck \& Eysenck, 1979, and Rogers et al., 1977). Of course, the argument presented here against a rating-time explanation for the superior recall of the recency-of-perceptual-contact condition would be strengthened if we could demonstrate that item-rating latencies do not affect the recall of words rated on the recency-of-perceptual-contact dimension. Experiment 4 addressed this issue and other issues.

Another question of some concern was whether our

Table 4

Mean Number of Words Rated (High T-L Frequency, Low T-L Frequency, Combined) and Mean Proportion of Words Recalled for Temporal Categories in the Recency-of-Perceptual-Contact Condition in Experiment 3

\begin{tabular}{lrrrrrrr}
\hline & \multicolumn{9}{c}{ Temporal Category } \\
\cline { 2 - 7 } Dependent Measure & Minutes & Hours & Days & Weeks & Months & Years & Never \\
\hline High T-L Frequency & 10.19 & 4.31 & 4.25 & 2.25 & 2.25 & 0.56 & 0.12 \\
Low T-L Frequency & 3.43 & 2.13 & 4.25 & 4.69 & 4.69 & 3.38 & 1.50 \\
Combined & 13.62 & 6.44 & 8.50 & 6.94 & 6.94 & 3.94 & 1.62 \\
Recall & .666 & .431 & .414 & .418 & .387 & .443 \\
\hline
\end{tabular}


replication of Nesbitt and Allen's (Note 1) observation of an inverse relationship between recall and temporal category (see Experiment 1) was an artifact of the distribution of rating responses. To this end, we examined the relationship between rating frequencies and temporal category and the relationship between recall and temporal category for the subjects serving in the recency-ofperceptual-contact condition. Table 4 shows the distribution of rating frequencies for high-frequency words, low-frequency words, and combined words as a function of temporal category. Clearly, the high-frequency words shifted the distribution in the direction of recency and produced a combined distribution that is markedly different from the distribution in Experiment 1 (see Table 1). It is important to note that this distribution of ratings precludes subjects' producing an inverse relationship between recall and temporal category by recalling approximately the same number of words from each category.

The proportions correct under the various temporal categories were subjected to a one-way within-subjects analysis of variance. Two of the 18 subjects in the recency-of-perceptual-contact condition were not included in this analysis because they failed to rate words in one of the first six temporal categories; consequently, they were eliminated to balance subjects across temporal categories. Likewise, only the first six temporal categories were included in the analysis, because 5 of the remaining 16 subjects failed to rate words in the "never" category. However, words rated in the first six categories accounted for $96 \%$ of the temporal category ratings. The analysis revealed a significant effect of temporal category $[\mathrm{F}(5,75)=3.51, \mathrm{MSe}=.048, \mathrm{p}<.01]$. Table 4 shows the relationship between recall and temporal category, and, as in Experiment 1, the data show a recency effect. Post hoc analysis (Newman-Keuls) showed that recall for the "minutes" category was significantly larger than for all other temporal categories $(p<.05)$ and that none of the other recall differences were significant. It should be clear that these results cannot be explained as an artifact of the distribution of temporal category ratings. If subjects in the recency-ofperceptual-contact condition had recalled approximately the same number of words from each category, they would not have produced a recency effect. Thus, some other explanation for the recency effect must be considered. Experiment 4 considered explanations for the relationship between recall and temporal category.

To summarize, Experiment 3 replicated Experiment 2 in showing that rating the recency of perceptual contact produced better recall than did rating pleasantness and that rating pleasantness produced better recall than rating length. An analysis of rating-task latencies suggested that these recall differences were not due to differences in rating (study or rehearsal) times, thereby suggesting that the results were due to qualitative differences in encoding elaboration. Further investigation of the inverse relationship between recall and temporal category showed that the effect could not be explained as an artifact of the distribution of temporal category ratings.

\section{EXPERIMENT 4}

Experiment 4 addressed two issues: (1) the relationship between item-rating latencies, item recall, and temporal category, and (2) the relationship between an assessment of autobiographical elaboration (i.e., episode recall) and item recall.

One potential explanation for the inverse relationship between recall and temporal category is that it is a studytime effect. That is, subjects may devote more time to rating the words they assign to early temporal categories (i.e., "minutes," "hours"), and this additional study or rehearsal produces better recall of these words. Rating latencies for individual words were collected to provide an empirical basis for testing the study-time explanation.

The second issue addresses one of the problems with research investigating elaboration, namely, that any wellremembered event can be too easily attributed to encoding elaboration. What is needed is to demonstrate a relationship between an assessment of encoding elaboration and recall. Consequently, we sought to demonstrate a relationship between autobiographical-episode recall and item recall. We suspected that, if subjects were not successful in retrieving an autobiographical episode as they rated an object label, they would substitute a "best guess" rating. For example, if subjects could not remember an autobiographical episode associated with the word "pincushion," they might guess that their last experience with a "pincushion" was several months or years ago. Words rated in this manner would not receive autobiographical elaboration, and, according to the autobiographical elaboration hypothesis, should not be recalled as well as words receiving autobiographical elaboration. To test this hypothesis, we assessed whether subjects' temporal ratings were based on the recall of autobiographical episodes. Following unexpected recall, the subjects were presented each word again and were asked to indicate whether their temporal rating was based on the recall of an autobiographical episode or an approximation. This allows us to calculate two conditional probabilities: (1) $\mathrm{P}$ (recall/yes)-the probability of recalling a word given that subjects reported basing their temporal ratings on the recall of autobiographical episodes, and (2) P(recall/no)-the probability of recalling a word given that subjects reported estimating their temporal ratings. The autobiographical elaboration hypothesis predicts $\mathrm{P}($ recall/yes $)>\mathrm{P}(\mathrm{recall} / \mathrm{no})$.

\section{Method}

Two studies were conducted by different experimenters. The replications differed in the following ways: (1) The time allowed for unexpected written free recall was $6 \mathrm{~min}$ in Study 1 and $10 \mathrm{~min}$ in Study 2, and (2) the number of subjects serving in each experiment was different. Because the results for both studies were substantially the same, the data were collapsed.

Fifty-one undergraduate students participated in the experiment. To achieve equal numbers of subjects across temporal 
categories, subjects who failed to use one of the first six temporal categories were eliminated (10 such subjects were eliminated). Three other subjects were eliminated: a foreign student, a subject who served in both studies (eliminated from the second study), and a subject who failed to use the "no" category during episode recall. Thus, the results are based on 38 subjects.

The subjects were run individually, seated before the keyboard and video monitor of an Apple II Plus microcomputer. All instructions, examples of the orienting task, and stimuli appeared in white uppercase print, centered on the black-andwhite video monitor (all via BASIC algorithm). The subjects received different random orderings of the 50 nouns used in Experiment 1.

The nouns were presented individually with the 7-point temporal-category scale (1-minutes, 2-hours, 3-days, 4-weeks, 5-months, 6-years, 7-never) centered under each noun. Words remained on the screen until they had been rated, and the presentation of the next word was nearly simultaneous with the keypress to rate the previous word. Thus, the rate of stimulus presentation was governed by the subjects' rate of rating.

The subjects rated the nouns by pressing the numeric key (1-7) corresponding to the appropriate temporal category. The latency of the rating response (time from stimulus presentation to keypress) was measured via a real-time clock (Mountain Hardware, Inc.) in the Apple computer to the nearest millisecond. The subjects were told that the experimenters were collecting normative data. They were informed that rating latencies were being collected, but were instructed as follows: "Note that even though your ratings are being timed, there is no need to rush. We are interested in how long it takes you to give your best estimate of recency of perceptual contact, not in how fast you can push a button."

Six (Study 1) or 10 (Study 2) minutes were allowed for unexpected written free recall. Following recall, the stimuli were presented individually on the video monitor in the same serial order as during the rating phase of the experiment. The subjects indicated whether they recalled an autobiographical episode during their temporal rating of the word by pressing "Y" for "yes" and "N" for "no." The following instructions accompanied this second rating task: "We would like to assess how many of your ratings were actually based on recalling an episode involving the object or a picture of the object named by a word. That is, we suspect that in some instances you were unable to recall an actual episode involving the word, and, consequently, you gave an educated guess concerning recency of perceptual contact. So we will present each word again and ask you to indicate whether you based your rating on the recall of an episode. It is very important that you answer these questions honestly. Press ' $Y$ ' for 'yes' and 'N' for 'no.'"

\section{Results and Discussion}

Table 5 shows the mean number of words rated and the mean number of episodes reported for each temporal category. The distribution of rating responses is very similar to the distribution of rating responses for Experiment 1 (see Table 1). The ratio of mean number of episodes reported to mean number of words rated shows the proportion of episodes recalled for each temporal category. These data show that the mean proportion of episodes recalled is inversely related to temporal category $($ minutes $=.933$, hours $=.836$, days $=.827$, weeks $=$ .643 , months $=.469$, years $=.457$ ) and suggests that a higher proportion of words rated in the early temporal categories received autobiographical elaboration.

The proportions correct for the first six temporal categories were subjected to a one-way within-subjects analysis of variance. The "never" category was not included in the analysis because 13 of the 38 subjects participating in the experiment failed to rate words in the "never" category. However, words rated in the first six temporal categories accounted for $93 \%$ of the temporal category ratings. The analysis showed a significant effect of temporal category $[F(5,185)=13.06$, $\mathrm{MSe}=.042, \mathrm{p}<.001]$. Post hoc analysis (NewmanKeuls) of the recall scores showed that the "minutes" and "hours" categories were not significantly different, that the "minutes" and "hours" categories were significantly larger than all other temporal categories $(p<.05)$, and that none of the other differences were significant. Thus, Experiment 4 replicated Experiments 1 and 3 in showing a recency effect (see Table 5).

The mean rating times (rounded to the nearest millisecond) for the first six temporal categories were also subjected to a one-way within-subjects analysis of variance. The analysis revealed a significant effect of temporal category $[\mathrm{F}(5,185)=12.78, \mathrm{MSe}=3,415,655.45$, $\mathrm{p}<.001]$. The results of this analysis also appear in Table 5 .

The data in Table 5 argue against a study-time explanation of the relationship between recall and temporal category. The study-time explanation would have received support if we had observed a direct relationship between rating time and recall, but Table 5 shows that rating time and recall are inversely related. The subjects clearly spent less time rating words that they assigned to recent temporal categories (i.e., "minutes" and "hours"), yet they showed better recall for these categories. This result is also inconsistent with a study-time or processingtime explanation of the superior recall of the recency-of-

Table 5

Mean Number of Words Rated, Mean Number of Episodes Reported, Mean Proportion of Words Recalled, Mean Rating Latency (in Milliseconds), and Mean P (Recall/Episode Reported) as a Function of Temporal Category in Experiment 4

\begin{tabular}{|c|c|c|c|c|c|c|c|}
\hline \multirow[b]{2}{*}{ Dependent Measure } & \multicolumn{7}{|c|}{ Temporal Category } \\
\hline & Minutes & Hours & Days & Weeks & Months & Years & Never \\
\hline Words Rated* & 4.03 & 5.79 & 10.63 & 8.03 & 10.21 & 7.95 & 3.32 \\
\hline Episodes Reported* & 3.76 & 4.84 & 8.79 & 5.16 & 4.97 & 3.63 & 0.82 \\
\hline Words Recalled* & .651 & .574 & .397 & .385 & .369 & .394 & \\
\hline Rating Latency* & 3766 & 4513 & 5149 & 5860 & 6581 & 6199 & \\
\hline P (Recall/Episode Reported)** & .691 & .621 & .401 & .518 & .422 & .476 & \\
\hline
\end{tabular}

${ }^{*} n=38 . \quad * *_{n}=31$. 
perceptual-contact condition noted in Experiments 1,2, and 3 . If processing time determines memory performance, then with qualitative differences in processing held constant, memory performance should be determined by the time taken to rate words. That is, within the recency-of-perceptual-contact condition, longer processing times should be associated with superior memory performance. Clearly, Table 5 shows just the opposite result.

To assess the relationship between episode recall and item recall, we calculated two conditional probabilities for each subject: (1) $\mathrm{P}$ (recall/yes)-the probability of recalling a word given that subjects reported basing their temporal ratings on the recall of autobiographical episodes, and (2) P(recall/no)-the probability of recalling a word given that subjects reported estimating their temporal ratings. Since the validity of these conditional probabilities depends on the accuracy with which the subjects remembered recalling autobiographical episodes during the temporal rating task, we sought to assess the subjects' episode-recall rating accuracy. Remember, the subjects rated words on a 7-point scale of recency of perceptual contact (1-minutes, 2-hours, 3-days, 4-weeks, 5-months, 6-years, 7-never), recalled the words (unexpected free recall), and then rated whether their temporal ratings were based on the recall of autobiographical episodes ( $\mathrm{Y}$-yes or $\mathrm{N}-\mathrm{no}$ ). Thus, an indirect indication of episode-recall rating accuracy is the percentage of words rated "never" or " 7 " during the temporal rating task that are rated "no" during the episode-recall rating task. That is, if the subjects correctly remembered what they did during the temporal rating of "never" words, they would respond "no" during the episode-recall rating of "never" words.

Twenty-five of the 38 subjects serving in the experiment assigned words to the "never" category during the temporal rating task. They assigned an average of five words to the "never" category, and their average episoderecall rating accuracy was $79.04 \%$. Fourteen of these subjects showed $100 \%$ episode recall accuracy; only five subjects had episode recall accuracies less than $66.6 \%$. Table 6 shows episode-recall rating accuracies across rating frequencies. These data suggest that subjects are fairly good at remembering whether their temporal ratings are based on the recall of autobiographical episodes.

The conditional probabilities were subjected to a oneway within-subjects analysis of variance. Remember, the autobiographical elaboration hypothesis predicts $\mathrm{P}($ recall $/$ yes $)>\mathrm{P}(\mathrm{recall} / \mathrm{no})$. The results showed that
$\mathrm{P}($ recall/yes) was significantly larger than $\mathrm{P}(\mathrm{recall} / \mathrm{no})$ [mean probabilities: .471 and .317 , respectively; $F(1,37)$ $=33.24, \mathrm{MSe}=.013, \mathrm{p}<.001]$. Indeed, 33 of the 38 subjects showed $\mathrm{P}($ recall/yes) $>\mathrm{P}(\mathrm{recall} / \mathrm{no})$ (sign test, $\mathrm{Z}=4.38, \mathrm{p}<.001)$. This result indicates that subjects' reports of autobiographical elaboration and recall are strongly related.

As an additional analysis, we calculated $\mathrm{P}$ (recall/yes) for each of the first six temporal categories for each subject and analyzed these data via a one-way withinsubjects analysis of variance. Subjects who failed to recall items in one of the six temporal categories were eliminated to achieve equal numbers of subjects across temporal categories; 7 subjects were eliminated, so the results are based on 31 subjects. The results (see Table 5) showed a significant effect of temporal category $[\mathrm{F}(5,150)=6.26, \mathrm{MSe}=.404, \mathrm{p}<.001]$. Post hoc analysis (Newman-Keuls) of the conditional recall scores revealed a recency effect: The "minutes" and "hours" categories were not significantly different; the "minutes" category was significantly larger than all other temporal categories $(p<.05)$; the "hours" category was not significantly different from the "weeks" category, but was significantly larger than all other temporal categories $(p<.05)$; and none of the other differences were significant. Also note that $\mathrm{P}($ recall $/$ yes $)>\mathrm{P}($ recall $/$ no $)$ across all six temporal categories in Table 5 .

Since there is little evidence for a study-time explanation of the relationship between recall and temporal category and little evidence that the recency effect is due to recall facilitated by environmental/situational cues (see Experiment 1), the conditional recall results suggest that the recency effect observed in Experiments 1,3 , and 4 is due to differences in autobiographical elaboration. Presumably, the autobiographical memories retrieved during the rating task differ in their richness or vividness, and richness or vividness varies inversely with temporal category ratings. Of course, before we can be sure of this explanation, future research will have to examine the relationship between autobiographical memory vividness, rating time, and recall.

\section{SUMMARY}

The experiments reported here demonstrate that autobiographical elaboration is a rich and powerful encoding process. Autobiographical orienting produced better noun recall than rating likelihood-of-recall $(\mathrm{Ex}$ -

Table 6

Mean Episode-Recall Rating Accuracy for Words Rated "Never" as a Function of the Number of Words Rated in the "Never" Category in Experiment 4

\begin{tabular}{|c|c|c|c|c|c|c|c|c|c|}
\hline & \multicolumn{9}{|c|}{ Number of Words Rated } \\
\hline & 1 & 2 & 3 & 4 & 5 & 6 & 7 & 10 & 14 \\
\hline Accuracy & 66.6 & 100 & 86.6 & 75.0 & 100 & 49.9 & 92.8 & 100 & 53.5 \\
\hline Number of Subjects & 3 & 4 & 5 & 2 & 1 & 4 & 2 & 2 & 2 \\
\hline
\end{tabular}


periment 1), pleasantness (Experiments 2 and 3), or length (Experiments 2 and 3). Furthermore, since there was little evidence that the superior recall produced by autobiographical elaboration was due to study or rehearsal time (Experiment 3), these experiments are further evidence that the qualitative nature of encoding processes has a substantial effect on human memory.

Unexpected free recall of words rated on the 7-point recency-of-perceptual-contact scale showed a recency effect; that is, recall varied inversely with temporal category (Experiments 1, 3, and 4). The recency effect appears to be due to differences in autobiographical elaboration (Experiment 4).

The results are consistent with an autobigraphical elaboration hypothesis: The more an orienting task elicits autobiographical memories, the better recall will be.

\section{REFERENCE NOTE}

1. Nesbitt, J. G., \& Allen, C. Memory for nouns as a function of perceived time elapsed between test and object-referent contact: Or, when was the last time you saw a pincushion? Paper presented at the meeting of the Southeastern Psychological Association, New Orleans, 1976.

\section{REFERENCES}

Christian, J., Bickley, W., Tarka, M., \& Clayton, K. Mea- sures of free recall of 900 English nouns: Correlations with imagery, concreteness, meaningfuiness, and frequency. Memory \& Cognition, 1978, 6, 379-390.

Craik, F. I. M., \& Tulving, E. Depth of processing and the retention of words in episodic memory. Journal of Experimental Psychology: General, 1975, 104, 268-294.

Chovitz, H. F., \& Schiffman, H. Frequency of episodic memories as a function of their age. Bulletin of the Psychonomic Society, 1974, 4, 517-518.

EysenCK, M. W., \& Eysenck, M. C. Processing depth, elaboration of encoding, memory stores, and expended processing capacity. Journal of Experimental Psychology: Human Learning and Memory, 1979, 5, 472-484.

GaLton, F. Inquiries into human faculty and its development (2nd ed.). New York: Dutton, 1911.

Packman, J. L., \& Battig, W. F. Effects of different kinds of semantic processing on memory for words. Memory \& Cognition, 1978, 6, 502-508.

Paivio, A., Yuille, J. C., \& Madigan, S. Concreteness, imagery, and meaningfulness values for 925 nouns. Journal of Experimental Psychology Monograph, 1968, 76(1, Pt. 2).

Rosinson, J. A. Sampling autobiographical memory. Cognitive Psychology, 1976, 8, 578-595.

Rogers, T. B., Kuiper, N. A., \& Kirker, W. S. Self-reference in the encoding of personal information. Journal of Personality and Social Psychology, 1977, 35, 677-688.

THORNDIKE, E. L., \& LORGE, I. The teacher's word book of 30,000 words. New York: Bureau of Publications, Teacher's College, Columbia University, 1944.

(Manuscript received December 8, 1982; revision accepted for publication June 20,1983 .) 